\title{
ARTICLE
}

\section{Conjugate vaccine produces long-lasting attenuation of fentanyl vs. food choice and blocks expression of opioid withdrawal-induced increases in fentanyl choice in rats}

\author{
E. Andrew Townsend ${ }^{1}$, Steven Blake ${ }^{2}$, Kaycee E. Faunce ${ }^{1}$, Candy S. Hwang ${ }^{2,3}$, Yoshihiro Natori ${ }^{2,4}$, Bin Zhou ${ }^{2}$, Paul T. Bremer ${ }^{2}$, \\ Kim D. Janda ${ }^{2}$ and Matthew L. Banks (D)
}

The current opioid crisis remains a significant public health issue and there is a critical need for biomedical research to develop effective and easily deployable candidate treatments. One emerging treatment strategy for opioid use disorder includes immunopharmacotherapies or opioid-targeted vaccines. The present study determined the effectiveness of a fentanyl-tetanus toxoid conjugate vaccine to alter fentanyl self-administration using a fentanyl-vs.-food choice procedure in male and female rats under three experimental conditions. For comparison, continuous 7-day naltrexone (0.01-0.1 mg/kg/h) and 7-day clonidine $(3.2-10 \mu \mathrm{g} / \mathrm{kg} / \mathrm{h})$ treatment effects were also determined on fentanyl-vs.-food choice. Male and female rats responded for concurrently available $18 \%$ diluted Ensure ${ }^{\circledR}$ (liquid food) and fentanyl (0-10 $\mu \mathrm{g} / \mathrm{kg} /$ infusion) infusions during daily sessions. Under baseline and saline treatment conditions, fentanyl maintained a dose-dependent increase in fentanyl-vs.-food choice. First, fentanyl vaccine administration significantly blunted fentanyl reinforcement and increased food reinforcement for 15 weeks in non-opioid dependent rats. Second, surmountability experiments by increasing the unit fentanyl dose available during the self-administration session 10-fold empirically determined that the fentanyl vaccine produced an approximate 22-fold potency shift in fentanyl-vs.food choice that was as effective as the clinically approved treatment naltrexone. Clonidine treatment significantly increased fentanyl-vs.-food choice. Lastly, fentanyl vaccine administration prevented the expression of withdrawal-associated increases in fentanyl-vs.-food choice following introduction of extended $12 \mathrm{~h}$ fentanyl access sessions. Overall, these results support the potential and further consideration of immunopharmacotherapies as candidate treatments to address the current opioid crisis.

Neuropsychopharmacology (2019) 44:1681-1689; https://doi.org/10.1038/s41386-019-0385-9

\section{INTRODUCTION}

The United States opioid crisis has resulted in a $493 \%$ increase in the diagnosis of opioid use disorder (OUD) from 2010 to 2016 [1]. Unfortunately, those who misuse illicit opioids are increasingly likely to consume, either knowingly or unknowingly, potent synthetic opioids such as fentanyl. For example, reported fatal overdoses from fentanyl and other synthetic opioids totaled 19,413 in 2016, an increase of $646 \%$ compared to 2010 [2]. Limitations in both effectiveness and deployment with current Food and Drug Administration (FDA)-approved pharmacotherapies such as the partial MOR agonist buprenorphine and the opioid antagonist naltrexone [3, 4] highlight the need for both preclinical and clinical research to develop more effective and readily available OUD treatments to address the opioid crisis.

Recently, the National Institutes of Health described several scientific areas to focus efforts toward the development of novel OUD treatment strategies [5]. One strategy includes using opioidtargeted vaccines to elicit antibody production by the host's immune system that recognize and selectively block the passage of a specific opioid into the brain and peripheral organs/tissue (i.e., immunopharmacotherapies: [6-8]). Immunopharmacotherapies may offer several advantages over current FDA-approved OUD treatments [6]. First, anti-opioid antibodies have so far not been shown to interact with any known receptors or proteins, suggesting that undesirable effects should be minimal [9-11]. Second, because the anti-opioid antibodies do not enter the brain, this treatment strategy should not affect endogenous brain opioid signaling, unlike current FDA-approved treatments (e.g., naltrexone, buprenorphine, methadone). Third, anti-fentanyl antibodies may have long half-lives, which would be hypothesized to extended inter-treatment intervals and increase compliance. Finally, because the antibodies have a high degree of specificity for the target opioid, a vaccinated subject could be treated with other structurally dissimilar opioids for pain management (e.g., oxycodone) or combined with current FDA-approved OUD treatments (e.g., buprenorphine or naltrexone).

The aim of the present study was to determine the effectiveness and time course of a conjugate fentanyl- $T$ vaccine on intravenous (IV) fentanyl self-administration using a fentanyl-vs.-food choice procedure in male and female rats. Although preclinical studies

\footnotetext{
${ }^{1}$ Department of Pharmacology and Toxicology, Virginia Commonwealth University School of Medicine, Richmond, VA 23298, USA; ${ }^{2}$ Departments of Chemistry and Immunology

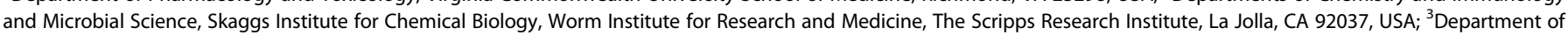

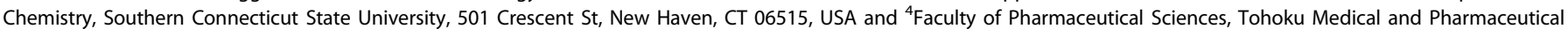
University, Komatsushima 4-4-1, Aoba-ku, Sendai 981-8558, Japan

Correspondence: Kim D. Janda (kdjanda@scripps.edu) or Matthew L. Banks (mbanks7@vcu.edu)
}

Received: 3 January 2019 Revised: 19 February 2019 Accepted: 1 April 2019

Published online: 2 May 2019 
have previously shown opioid-targeted vaccines to reduce rates of opioid self-administration [12-15], there are no published studies examining whether opioid-targeted vaccines would not only decrease opioid-taking behaviors, but also increase behavior directed towards more adaptive nondrug reinforcers, consistent with OUD treatment goals $[16,17]$. Thus, a fentanyl-vs.-food choice procedure was used because preclinical choice procedures have increasingly shown translational concordance to both human laboratory studies and double-blind placebo-controlled clinical trials in the evaluation of candidate medications for substance use disorders [18-20]. Two experimental conditions were examined. First, vaccine effectiveness to attenuate fentanyl-vs.-food choice was examined under daily 2 -h fentanyl access conditions. Continuous 7-day naltrexone treatment was utilized as a positive control because naltrexone is FDA approved for OUD in nondependent individuals [21, 22]. Continuous 7-day treatment with the $a_{2}$ adrenergic agonist clonidine was selected as an active negative control. Clonidine will relieve some somatic opioid withdrawal signs in opioid-dependent OUD patients and has been shown to decrease stress-induced reinstatement in rats as well as reduce the risk of relapse when used as an adjunct to buprenorphine in humans [23-25]. However, clonidine does not prevent relapse when used as a monotherapy [26]. In a second series of experiments, the vaccine was evaluated for its ability to prevent withdrawal-associated increases in fentanyl-vs.-food choice following extended ( $12 \mathrm{~h} /$ day) fentanyl access. A previous study showed that $12 \mathrm{~h}$ extended fentanyl access produced opioid dependence as demonstrated by naloxone-precipitated somatic withdrawal signs [27]. Furthermore, opioid withdrawal will also increase opioid vs. non-opioid choice in both monkeys and rats $[28,29]$. In addition, these withdrawal-associated increases in opioid choice have been implicated as one critical driver of opioid abuse and hypothesized to be an important target for OUD treatment development [30-32].

\section{MATERIALS AND METHODS}

\section{Subjects}

A total of 19 Sprague-Dawley rats (9 male, 10 female) were acquired at 10 weeks of age (Envigo Laboratories, Frederick, MD, USA) and surgically implanted with vascular access ports (Instech, Plymouth Meeting, PA) and custom-made jugular catheters as described previously [33]. Rats were singly housed in a temperature and humidity-controlled vivarium that was maintained on a 12-h light/dark cycle (lights off at 1800 hours). Water and food (Teklad Rat Diet, Envigo) were provided ad-libitum in the home cage. Behavioral testing was conducted five days per week from approximately $1400-1600$ hours unless otherwise noted. Rat maintenance and research were conducted in accordance with the 2011 guidelines for the care and use of laboratory animals and protocols were approved by the Virginia Commonwealth University Institutional Animal Care and Use Committee.

Self-administration apparatus and catheter maintenance

Twelve modular operant chambers located in sound-attenuating cubicles (Med Associates, St. Albans, VT) were equipped with two retractable levers, a set of three LED lights (red, yellow, green) mounted above each lever, and a retractable cup $(0.1 \mathrm{ml})$ located between the levers for delivering diluted liquid food $(18 \% \mathrm{v} / \mathrm{v}$ vanilla flavor Ensure ${ }^{\circledR}$ in tap water; Abbott Laboratories, Chicago, IL). Intravenous (IV) fentanyl was delivered by a syringe pump (PHM-100, Med Associates) located inside the sound-attenuating cubicle as described previously [34]. After each behavioral session, catheters were flushed with gentamicin $(0.4 \mathrm{mg})$ followed by 0.1 $\mathrm{ml}$ of heparinized saline $(30 \mathrm{U} / \mathrm{ml})$. Catheter patency was verified periodically and at study end by instantaneous muscle tone loss following IV methohexital $(0.5 \mathrm{mg})$ administration.
Fentanyl vs. food choice

After stable responding for IV fentanyl (3.2 $\mu \mathrm{g} / \mathrm{kg} / \mathrm{infusion})$ and liquid food (18\% diluted Ensure $\left.{ }^{\circledR}\right)$ alone, rats were trained to respond under a fentanyl-vs.-food choice procedure modified from drug-vs.-food choice procedures in rats [35] and monkeys $[31,36,37]$. The behavioral session consisted of five 20-min response components each preceded by a 4-min "sample" component. Each sample component started with a noncontingent infusion of the unit fentanyl dose available during the subsequent response component followed by a 2-min time out. Next, a 5-s presentation of liquid food was programmed followed by a 2-min time out. Following this second time out, the response component would begin. During each response component, both levers were extended, a red stimulus light above the left lever was illuminated to signal liquid food availability and a green stimulus light above the right lever was illuminated to signal IV fentanyl availability. Response requirement (fixed-ratio; FR5) completion on the left lever resulted in a 5-s presentation of liquid food whereas response requirement (FR5) completion on the right lever resulted in the delivery of the IV fentanyl dose available for that component. Responding on one lever reset the ratio requirement for the other lever. The liquid food concentration was constant throughout the session. A different fentanyl dose was available during each of the five successive response components $(0,0.32,1.0,3.2$, and $10 \mu \mathrm{g} / \mathrm{kg} / \mathrm{inf}$ during components $1-5$, respectively). Fentanyl dose was varied by changing the infusion duration (e.g. $300 \mathrm{~g}$ rat: $0,0.5,1.56,5$, and $15.6 \mathrm{~s}$ during components $1-5$, respectively) and the green light above the fentanyl-lever flashed on and off in $3 \mathrm{~s}$ cycles (i.e. longer flashes associated with larger fentanyl doses).

During each response component, rats could complete up to 10 total ratio requirements between the food- and fentanylassociated levers. Each ratio requirement completion initiated a 20-s time out, the retraction of both levers, and extinction of the red and green stimulus lights. If all 10 ratio requirements were completed before $20 \mathrm{~min}$ had elapsed, then both levers retracted, and stimulus lights were extinguished for the remainder of that component. Choice was considered stable when the smallest unit fentanyl dose that maintained at least $80 \%$ of completed ratio requirements on the fentanyl-associated lever was within a 0.5 log unit of the running mean for three consecutive days with no trends. Once these criteria were met, choice testing occurred Monday through Thursday each week unless otherwise specified.

\section{Warm water tail withdrawal}

A water bath (Precision, 280 Series Water Bath, Winchester, VA) was maintained at $50^{\circ} \mathrm{C}\left( \pm 1^{\circ} \mathrm{C}\right)$. Each session began by gently wrapping the rat with a towel, leaving the tail exposed. The distal five $\mathrm{cm}$ of the tail was immersed in heated water $\left(50^{\circ} \mathrm{C}\right)$ and the latency to fully remove the tail was recorded with a digital chronograph with a $0.01 \mathrm{~s}$ resolution (Sports Timer, Fisher Brand, Hampton, $\mathrm{NH}$ ). If the rat did not remove its tail by $20 \mathrm{~s}$, the experimenter removed the tail and a latency of $20 \mathrm{~s}$ was assigned. Following baseline latency determination, IV saline or fentanyl was administered followed by a $0.1 \mathrm{ml}$ saline flush and tail-withdrawal latency was redetermined $60 \mathrm{~s}$ later. The pretreatment time was based on published IV fentanyl time-course data [38]. Saline was always injected first, followed by cumulative fentanyl doses $(1-1000 \mu \mathrm{g} / \mathrm{kg})$ administered in $0.5 \mathrm{log}$ dose increments. Testing continued until a maximum latency of $20 \mathrm{~s}$ was observed. Tail-withdrawal test sessions occurred each Friday unless otherwise specified.

\section{Fentanyl vaccine experiments}

Following baseline data collection (i.e., M-Th, choice testing; F, tail withdrawal; Week 0), six rats (three males, three females) were vaccinated on Monday at weeks 1 and 3 (Timeline: Fig. 2a). Fentanyl hapten was conjugated to $\Pi T$ as previously described [39] 
and solubilized in $50 \%$ glycerol, $50 \%$ phosphate-buffered saline. Fentanyl hapten copies were approximately 23 per protein based on a bovine serum albumin (BSA) surrogate $[9,39]$. On a per rat basis, $250 \mu \mathrm{g}$ conjugate fentanyl-TT hapten was mixed with $50 \mu \mathrm{g}$ phosphorothioate-modified CpG ODN 1826 (Eurofins Genomics, Louisville, KY) and $0.75 \mathrm{mg}$ alhydrogel adjuvant 2\% (InvivoGen, San Diego, CA) for $30 \mathrm{~min}$ and then refrigerated $24 \mathrm{~h}$ prior to IP administration. Every two weeks after the initial vaccination, tail vein blood was collected under isoflurane anesthesia into lithium heparin tubes (BD, Franklin Lakes, NJ). Tubes were immediately centrifuged at 3300 RPM for 15 min (Ample Scientific, Model E331, Norcross, GA) and serum was pipetted into microcentrifuge tubes and stored at $-80^{\circ} \mathrm{C}$ until analyzed. Anti-fentanyl titer and affinity measurements were performed as previously described [39]. Rats did not participate in behavioral tests on days when either fentanyl vaccine administration or blood collection occurred, resulting in an abbreviated testing week (i.e., T-Th, choice testing; $F$, tail withdrawal).

To determine the robustness of fentanyl vaccine effectiveness, two additional experiments were conducted. First, the four rats that maintained catheter patency at week 17 were vaccinated ( 375 $\mu \mathrm{g}$ of conjugate fentanyl-TT hapten $+50 \mu \mathrm{g}$ CpG ODN $1826+0.75$ mg alhydrogel adjuvant 2\%) six hours after a Friday fentanyl tailwithdrawal test. Two days later, the four vaccinated rats (two male, two female) and six nonvaccinated rats (three male, three female; not used elsewhere) were allowed to self-administer fentanyl $(3.2 \mu \mathrm{g} / \mathrm{kg} /$ infusion) under an FR5, $10 \mathrm{~s}$ time out schedule of reinforcement during daily 12 -h sessions from 1800 to 0600 hours 5 days per week for 14 days in addition to the daily 2 -h fentanylvs.-food choice sessions from 1400 to 1600 hours (i.e., preceding $\mathrm{M}-\mathrm{F}$ choice sessions). The six nonvaccinated rats had an average (SEM) of 10.8 (1.2) weeks of fentanyl self-administration history under the choice procedure before this experiment. During these extended access sessions, fentanyl availability was signaled by the extension of the left lever and green stimulus light illumination above the left lever. During the time-out period, the stimulus light was extinguished, and the left lever was retracted.

Second, these four vaccinated rats (two male, two female) were tested in a single fentanyl-vs.-food choice session wherein the available unit fentanyl doses were increased 10-fold (i.e., $3.2-100 \mu \mathrm{g} / \mathrm{kg} /$ infusion) relative to the original dose range (i.e., $0.32-10 \mu \mathrm{g} / \mathrm{kg} /$ infusion). All other aspects of the choice procedure were identical. This test session occurred on Tuesday of week 21 and a tail-withdrawal test occurred on Friday.

Chronic naltrexone and clonidine treatment experiments

Following baseline fentanyl-vs-food choice and fentanyl antinociception determination, seven non-vaccinated rats $(3$ male, 4 female) were aseptically implanted with osmotic pumps (Alzet, Model 2001, Cupertino, CA) in the mid-scapular subcutaneous region under isoflurane anesthesia after a tail-withdrawal test. Following pump implantation, fentanyl-vs.-food choice and tailwithdrawal testing resumed on $\mathrm{M}$-Th and $\mathrm{F}$, respectively. After the tail-withdrawal test, rats were again anesthetized with isoflurane, and the pump was removed. Once fentanyl-vs.-food choice and fentanyl tail-withdrawal latencies returned to baseline levels, another osmotic pump was aseptically implanted. Treatment conditions were saline, naltrexone $(0.01,0.032$, $0.1 \mathrm{mg} / \mathrm{kg} / \mathrm{h})$, or clonidine $(3.2,10 \mu \mathrm{g} / \mathrm{kg} / \mathrm{h})$. The maximum naltrexone dose $(0.1 \mathrm{mg} / \mathrm{kg} / \mathrm{h})$ was based its saline solubility (i.e., approximately $40 \mathrm{mg} / \mathrm{ml}$ ) and the maximum clonidine dose $(10 \mu \mathrm{g} / \mathrm{kg} / \mathrm{h})$ was based on sustained lethargy in some rats. Saline and naltrexone were incorporated into a counterbalanced dosing order before the counterbalanced clonidine dose order.

Data analysis

For fentanyl-vs.-food choice studies, the primary dependent measures were percent fentanyl choice, defined as (number of ratio requirements, or 'choices', completed on the fentanylassociated lever/total number of choices completed on both levers) $\times 100$ and number of choices per component. Data were first averaged within a rat across $\mathrm{M}$-Th (or T-Th, if blood collection or vaccine administration occurred) and then averaged between rats to yield group mean data. Results were then plotted as a function of the fentanyl dose and analyzed using a mixed-model analysis (JMP Pro 12) with either vaccination week or treatment drug dose and fentanyl dose as the fixed main effects. A Dunnett's test was performed to compare treatment effects within a fentanyl dose. In addition, log $\mathrm{ED}_{50}$ values $( \pm 95 \%$ confidence limits) of percent fentanyl choice and choices per component were calculated using linear regression when at least three points were above and below the 50\% effect level or interpolation when only two data points were available. Furthermore, if percent fentanyl choice did not surpass $50 \%$ or if a $50 \%$ reduction in choices per component was not observed, a value of $100 \%$ or $0 \%$ was assigned to the next highest $0.5 \mathrm{log}$ dose for percent fentanyl choice or choices per component, respectively. This contingency was used to facilitate the conservative estimation of surmountability of the vaccine's effects on choice-related measures (i.e., Fig. 4), as the choice function of a single female rat was unaffected by the presentation of a 10-fold higher fentanyl unit dose range, exclusively choosing ten food presentations in all components.

Tail-withdrawal latencies were expressed as percent maximum effect (\%MPE) using the following equation: \%MPE = [(Test latency-saline latency $) /(20 s$-saline latency $)] \times 100$ where test latency was the latency after fentanyl administration, and saline latency was the latency after saline administration at experiment onset. Log \%MPE $\mathrm{ED}_{50}$ values $( \pm 95 \%$ confidence limits) were calculated as described above. A treatment was considered to be significantly different if $95 \%$ confidence limits did not overlap.

For the extended fentanyl access studies, the number of fentanyl infusions earned during the 12-h sessions were compared between vaccinated and non-vaccinated rats using a two-way ANOVA, with time as the within-subjects factor and vaccination status as the between-subjects factor. A significant interaction was followed by a Sidak post-hoc test to compare fentanyl infusions at each day between groups. Fentanyl choice data (i.e., percent fentanyl choice and choices per component) were categorized by their proximity to the extended access sessions (i.e., Day 1, Days 2-6, Days 9-13) and analyzed separately for vaccinated and nonvaccinated rats using a mixed-model analysis (JMP Pro 12) with extended access category and fentanyl dose as the fixed main effects. Following a significant interaction, a Dunnett's test was performed to compare treatment effects within a fentanyl dose to data collected during Day 1 . The criterion for significance was set a priori at the $95 \%$ confidence level $(p<0.05)$.

For the fentanyl choice surmountability experiment in the four rats (two males, two females) that maintained catheter patency, pre-vaccination log $\mathrm{ED}_{50}$ values ( $\pm 95 \%$ confidence limits) were compared to those obtained during vaccination week 21 on (1) percent fentanyl choice, (2) choices per component, and (3) \% MPE. Values were considered to be different if $95 \%$ confidence limits did not overlap.

Drugs

Fentanyl $\mathrm{HCl}$ and (-)-naltrexone $\mathrm{HCl}$ were provided by the National Institute on Drug Abuse Drug Supply Program (Bethesda, $\mathrm{MD}$ ) and dissolved in sterile saline. Clonidine $\mathrm{HCl}$ (Sigma Aldrich, St. Louis, MO) was purchased from commercial suppliers. Fentanyl, naltrexone, and clonidine solutions were passed through a $0.22-$ micron sterile filter (Millex GV, Millipore Sigma, Burlington, MA) before administration. All drug doses were expressed as the salt forms listed above and delivered based on weights collected weekly. 

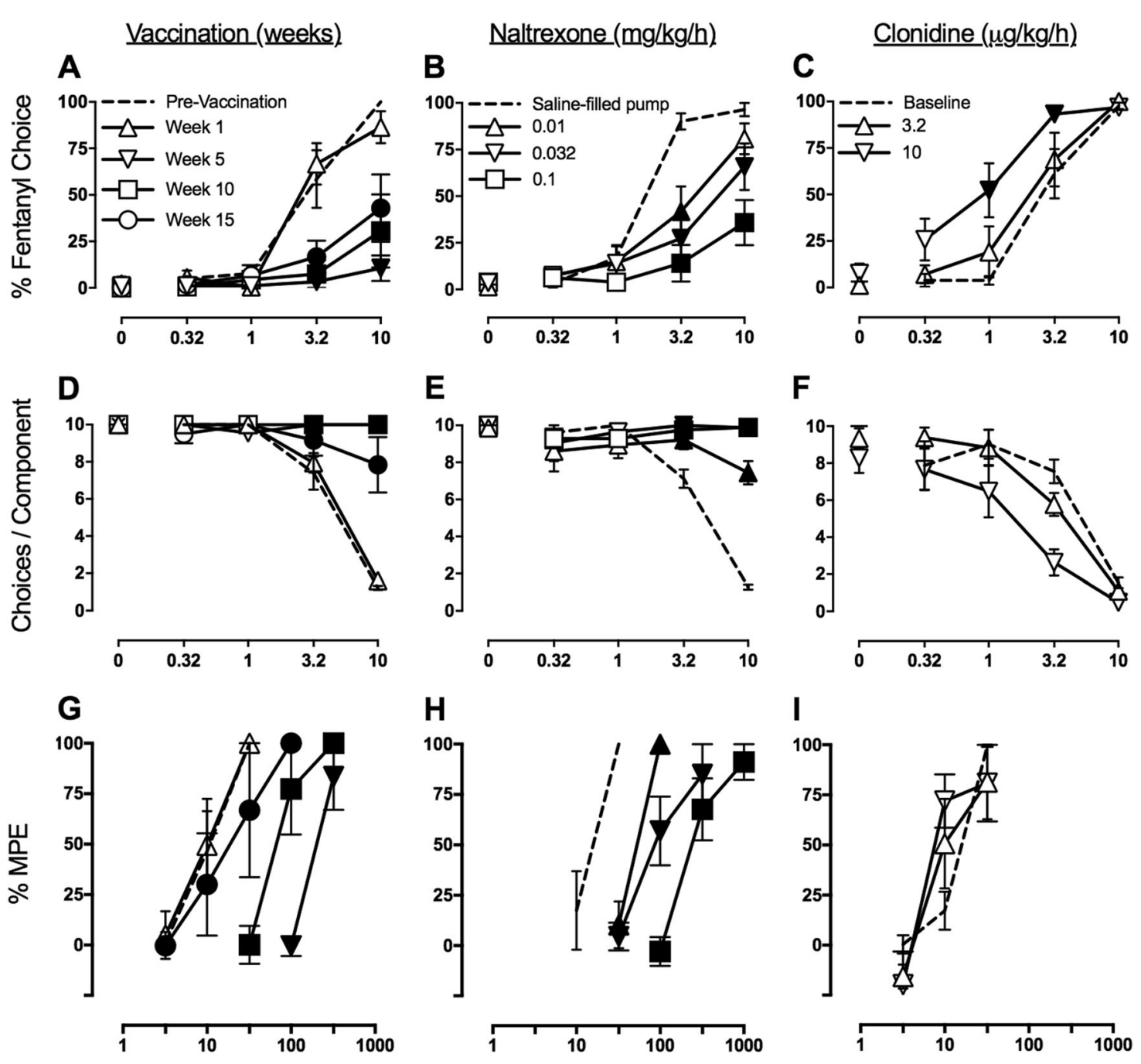

Fentanyl ( $\mu \mathrm{g} / \mathrm{kg}$, i.v.)

Fig. 1 Effectiveness of a fentanyl vaccine, repeated naltrexone (positive control), or repeated clonidine (negative control) to attenuate intravenous (IV) fentanyl-vs.-food choice (a-f) and IV fentanyl antinociception ( $(\mathbf{- i})$ in male and female rats. Abscissa: IV fentanyl dose in $\mu \mathrm{g} / \mathrm{kg}$ (unit dose for a-f, cumulative dose for $\mathbf{g}-\mathbf{i}$ ). Top row ordinates: Percentage of completed ratio requirements on the fentanyl-associated lever. Center row ordinates: Number of choices completed per component. Bottom row ordinates: Percent maximum possible effect (\%MPE) collected in a warm water tail-withdrawal procedure. a, d, g: Selected timepoints following conjugate fentanyl vaccine administration: Prevaccination (Week 0), Week 1, and Week 5: $n=3$ males, 3 females; Week 10 and Week 15: $n=2$ males, 2 females. b, e, h: Chronic 7-day treatment with naltrexone- or saline-filled pumps: $n=3$ males, 4 females. c, $\mathbf{f}$, i: Chronic 7-day treatment with clonidine-filled pumps relative to data averaged from the weeks preceding pump implantation: $n=2$ males, 4 females. Points represent mean \pm SEM and filled symbols denote significant difference relative to baseline, defined as $p<0.05$ in choice-related measures and non-overlapping $95 \%$ confidence limits for warm water tail-withdrawal related measures

\section{RESULTS}

Baseline fentanyl-vs.-food choice and fentanyl antinociception Under baseline conditions, liquid food was chosen over no or small ( 0.32 and $1 \mu \mathrm{g} / \mathrm{kg} /$ infusion) fentanyl doses (dashed lines; Fig. $1 \mathrm{a}-\mathrm{c})$. As the fentanyl dose increased, behavior was reallocated to the fentanyl lever and the largest fentanyl dose $(10 \mu \mathrm{g} / \mathrm{kg} /$ infusion) maintained near exclusive fentanyl choice. Additionally, choices per component decreased as a function of increasing fentanyl doses (dashed lines; Fig. 1d-f). The bottom panels (dashed lines; Fig. 1g-i) show baseline fentanyl antinociception and a cumulative dose of $32 \mu \mathrm{g} / \mathrm{kg}$ produced $100 \%$ MPE in all rats. Raw tail-withdrawal latencies $( \pm$ SEM) after IV saline were $10.2 \mathrm{~s}( \pm 0.4)$ and $7.4 \mathrm{~s}( \pm 1.0)$ for the vaccine and naltrexone/ clonidine treatment groups, respectively. No baseline group differences were detected for any endpoint.
Vaccine effects on fentanyl choice and thermal antinociception Vaccine administration produced a significant and sustained decrease in fentanyl choice and reciprocal increase in food choice from weeks 5 to 15 (Fig. 1a: fentanyl dose: $F_{4,101}=45, p<0.001$; interaction: $\left.F_{16,101}=7.6, p<0.001\right)$. In addition, choices completed during the last two components significantly increased, with rats choosing liquid food over fentanyl to a greater extent compared to baseline (Fig. 1d: fentanyl dose: $F_{4,99}=120, p<$ 0.001 ; interaction: $\left.F_{16,99}=28, p<0.001\right)$. Results separated by sex are shown in Supplementary Fig. 2.

Vaccine administration also significantly decreased fentanyl antinociceptive potency up to a maximum 24-fold potency shift at week 5 (Fig. 1g). Fentanyl antinociceptive potency time course is shown in Fig. $2 \mathrm{~b}$ and Supplementary Table 1 shows weekly potency shifts for the entire experimental period. Figure $2 c$ shows 


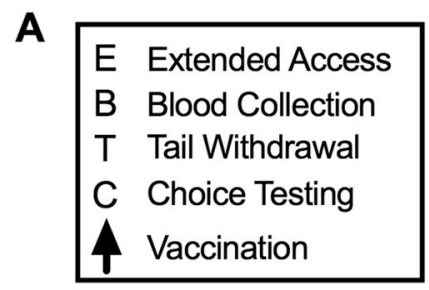

\section{EE}

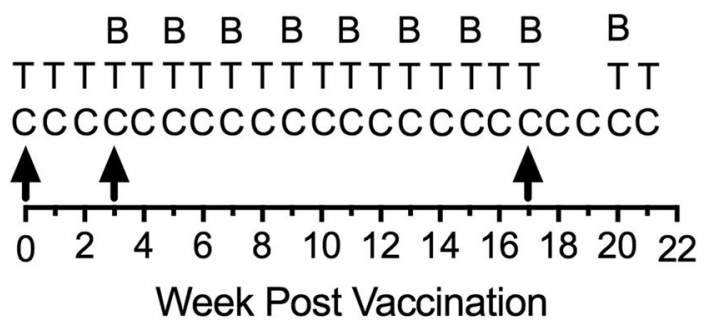

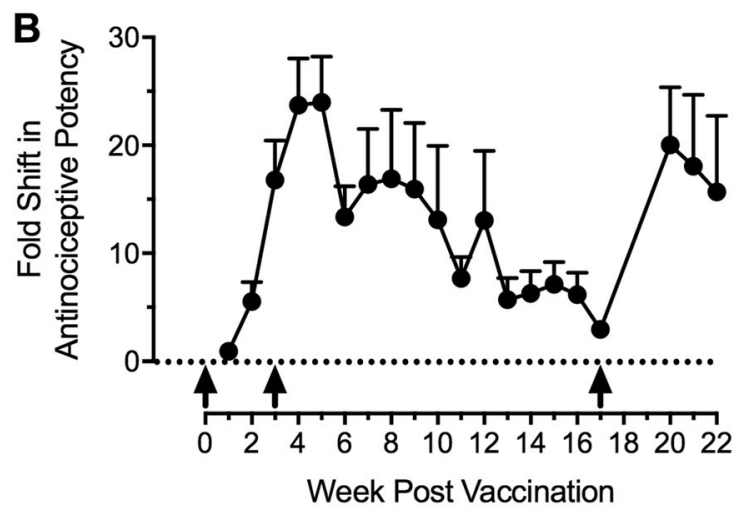
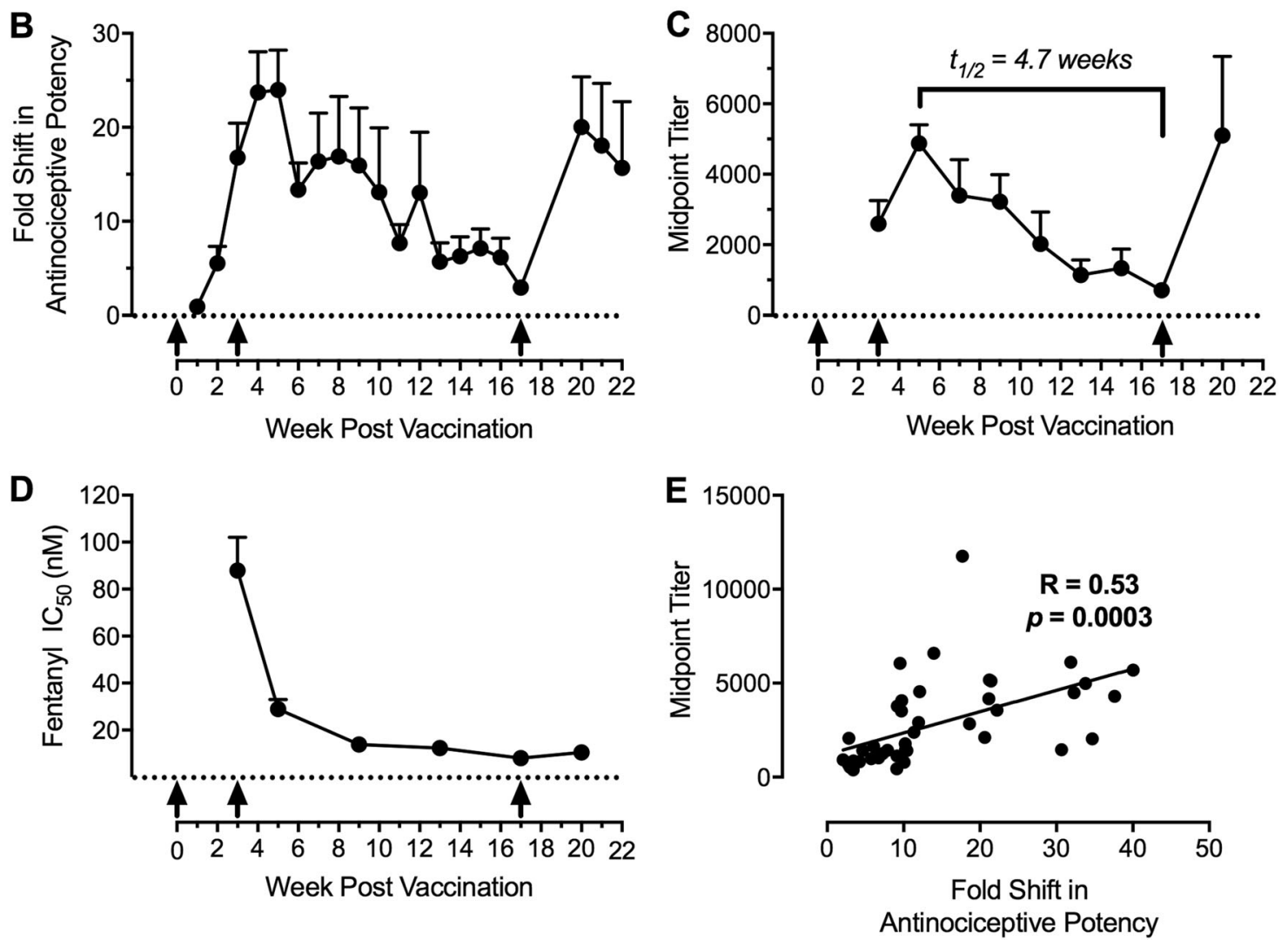

Fig. 2 Fentanyl antinociception potency shifts, midpoint titer levels and antibody affinity over the entire 20-week experimental period. A timeline of experimental procedures is depicted in $\mathbf{a}$. $\mathbf{b}$ shows fentanyl vaccine effects on fentanyl antinociceptive potency as a function of experimental week. Abscissa: timeline with arrows indicating dates of vaccination. Ordinate: fold shift in fentanyl antinociceptive potency relative to pre-vaccination $\mathrm{ED}_{50}$ values. c shows anti-fentanyl midpoint titers as a function of experimental week. Abscissa: timeline with arrows indicating dates of vaccination. Ordinate: Midpoint titer levels. $\mathbf{d}$ shows anti-fentanyl antibody affinity as a function of experimental week. Abscissa: timeline with arrows indicating dates of vaccination. Ordinate: Fentanyl IC 50 values (nM) determined from antiserum. e shows the correlation between midpoint tier levels and fold shifts in fentanyl antinociceptive potency $(\mathrm{R}=0.53, p=0.003)$. Up to Week 6 of vaccination schedule: $n=3$ males, 3 females; Weeks 7-8: $n=3$ males, 2 females; Weeks 9-20: $n=2$ males, 2 females. With the exception of e, points represent mean \pm SEM

midpoint titer levels peaked at week 3 and then decayed, resulting in a half-life of 4.7 weeks $\left(95 \% \mathrm{CL}=3.7-6.5, R^{2}=0.96\right)$. Midpoint titer levels rebounded three weeks later to previously observed peak levels following the third vaccine booster. Figure $2 \mathrm{~d}$ illustrates the progression of antibody-fentanyl affinity $\left(\mathrm{IC}_{50}\right.$ values) over time. Antibody affinity to fentanyl peaked by week 17 (8.1 nM). Moreover, by week 8 antibody-fentanyl affinity was basically maximized. Finally, midpoint titer levels and fentanyl antinociception potency shifts were positively correlated (Fig. 2e: $\mathrm{R}=0.53, p=0.0003$ ). Results separated by sex are shown in Supplementary Fig. 2.

Naltrexone effects on fentanyl choice and thermal antinociception All three naltrexone doses significantly decreased fentanyl choice compared to saline (1B: fentanyl dose: $\mathrm{F}_{4,24}=61, p<0.0001$; naltrexone dose: $F_{3,18}=10, p=0.0004$; interaction: $F_{12,72}=10.2$, $p<0.001)$. In addition, all naltrexone doses significantly increased choices completed per component ( $1 \mathrm{E}$ : fentanyl dose: $\mathrm{F}_{4,24}=10$, $p<0.0001$; naltrexone dose: $F_{3,18}=25, p<0.001$; interaction: $F_{12,} \quad 72=40, p<0.001$ ). Finally, naltrexone also significantly decreased fentanyl antinociceptive potency and the largest naltrexone dose $(0.1 \mathrm{mg} / \mathrm{kg} / \mathrm{h})$ tested produced an approximate 24-fold potency shift $(1 \mathrm{H})$. Supplementary Table 1 reports fentanyl potency shifts during naltrexone treatment.

Clonidine effects on fentanyl choice and thermal antinociception Clonidine $(10 \mu \mathrm{g} / \mathrm{kg} / \mathrm{h})$ significantly increased fentanyl choice compared to the pre-pump baseline ( $1 \mathrm{C}$ : fentanyl dose: $\mathrm{F}_{4,75}=$ $41, p<0.0001$; interaction: $\left.\mathrm{F}_{8,}, 75=2.9, p=0.008\right)$. However, clonidine did not alter choices completed per component (1 F: 

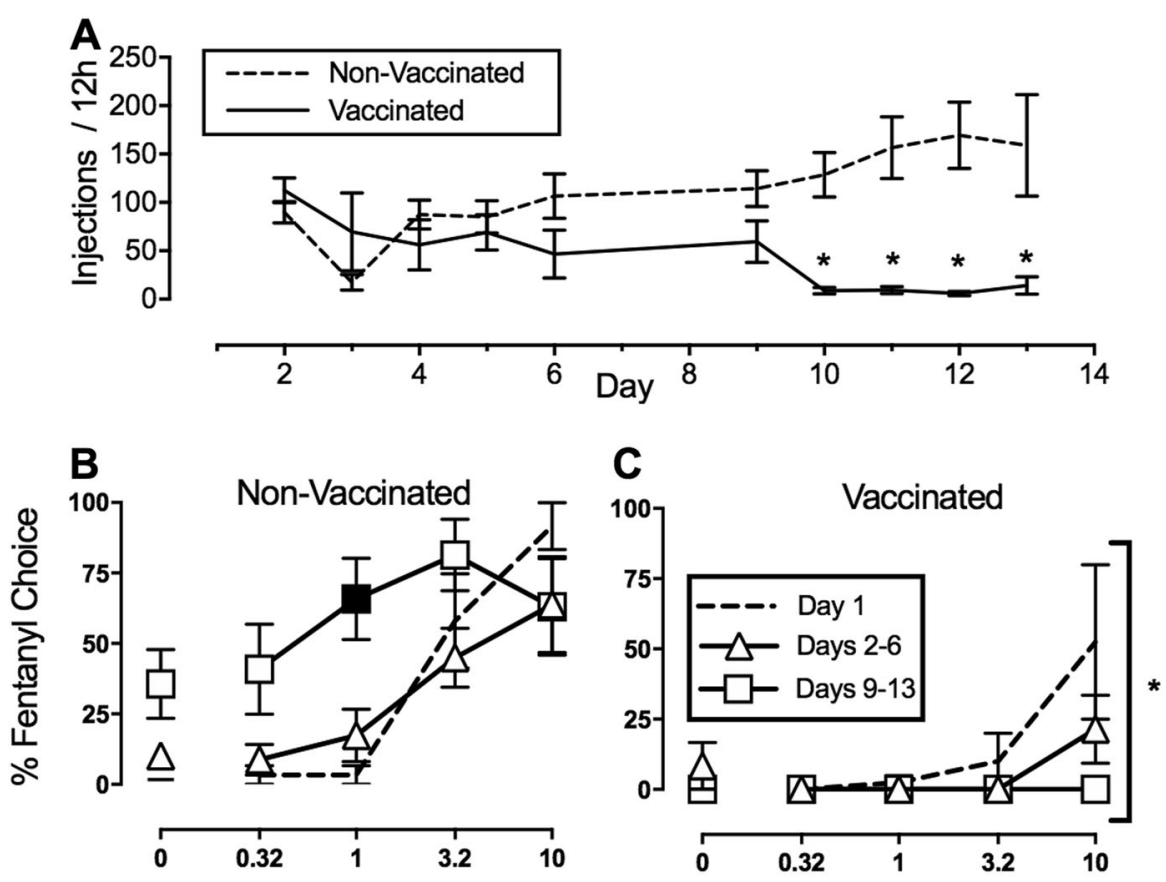

D

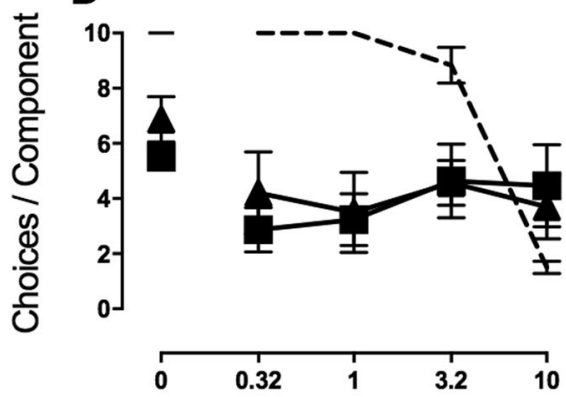

E
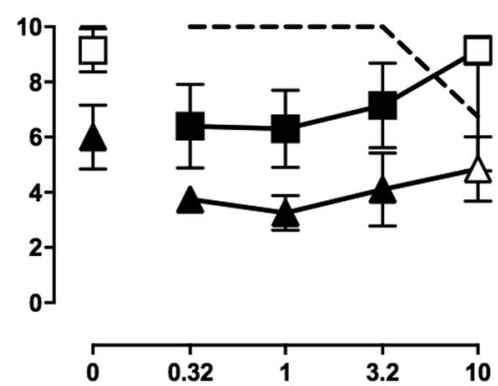

Fentanyl $(\mu \mathrm{g} / \mathrm{kg}$, i.v.)

Fig. 3 Conjugate fentanyl vaccine administration blocked opioid dependence and withdrawal-associated increases in fentanyl choice produced by extended fentanyl self-administration sessions. a shows rates of fentanyl self-administration during the $12 \mathrm{~h}$ extended-access session in vaccinated (solid line: $n=2$ males, 2 females; tested in weeks 18-19 of vaccination schedule following the final week 17 booster injection) and non-vaccinated (dashed line: $n=3$ males, 3 females). Abscissa: experimental day. Ordinate: Number of fentanyl infusions (3.2 $\mu \mathrm{g} / \mathrm{kg}$ unit dose) during each $12-\mathrm{h}$ session. ${ }^{*}$ indicates difference between vaccinated and non-vaccinated rats at a day ( $\left.p<0.05\right)$. $\mathbf{b}-\mathbf{e}$ shows fentanyl vs. food choice results assessed $8 \mathrm{~h}$ after extended access sessions in non-vaccinated (left) and vaccinated (right) rats. Abscissa: intravenous unit fentanyl dose in $\mu \mathrm{g} / \mathrm{kg} /$ infusion. Middle ordinates $(\mathbf{b}, \mathbf{c})$ : Percentage of completed ratio requirements on the fentanylassociated lever. Bottom ordinates (d, e): Number of choices completed per component. Points represent mean \pm SEM and filled symbols denote significant difference at a unit fentanyl dose relative to Day 1 , defined as $p<0.05 .{ }^{*}$ indicates significant $(p<0.001)$ interaction between fentanyl dose and condition

fentanyl dose: $\left.F_{4,79}=21, p<0.0001\right)$ or fentanyl antinociceptive potency (1I). Supplementary Table 1 reports fentanyl potency shifts during clonidine treatment.

Fentanyl vaccine effects on extended fentanyl self-administration and fentanyl choice

Figure $3 a$ shows rates of fentanyl self-administration during 12-h extended sessions in non-vaccinated (dashed line, $n=6$ ) and vaccinated (solid line, weeks $18-19$ of vaccination schedule, $n=4$ ) rats after the week 17 vaccine booster. Both groups of rats earned approximately 100 infusions during the first 12-h extended session. However, the number of fentanyl infusions increased over the 14-day experimental period in the non-vaccinated group and significantly decreased to approximately ten fentanyl infusions in the vaccinated group (vaccination status: $F_{1,8}=6$, $p=0.04$; interaction: $\mathrm{F}_{9,72}=7.9, p<0.001$ ). Figure $3 \mathrm{~b}$ (nonvaccinated) and $3 C$ (vaccinated) depict fentanyl choice data collected either $2 \mathrm{~h}$ before the first extended access session (Day 1 , dashed line), $8 \mathrm{~h}$ post the first five extended access sessions (Days 2-6, triangles), or second five extended access sessions (Days 9-13, squares). Extended access sessions produced withdrawal-associated increases in fentanyl choice in nonvaccinated rats, such that fentanyl choice was significantly increased for $1 \mu \mathrm{g} / \mathrm{kg} /$ infusion fentanyl during Days 9-13 compared to Day 1 (3B: condition: $\mathrm{F}_{2,137}=3.3, p=0.04$; fentanyl dose: $\mathrm{F}_{4,137}=32, p<0.0001$; interaction: $\left.\mathrm{F}_{8,137}=5.5, p<0.001\right)$. In contrast, fentanyl choice decreased in the vaccinated rats $(3 \mathrm{C}$ : fentanyl dose: $\mathrm{F}_{4,101}=45, p<0.001$; interaction: $\mathrm{F}_{16,101}=7.6, p<$ 0.001 ), recapturing vaccine effectiveness shown in Fig. $2 a$. Extended fentanyl access significantly decreased choices completed during early choice components (1-4) and significantly increased choices completed during $10 \mu \mathrm{g} / \mathrm{kg} /$ infusion fentanyl availability in non-vaccinated rats (3D: condition: $\mathrm{F}_{4,20}=9.3, p=$ 0.0002 ; fentanyl dose: $F_{2,10}=15, p=0.001$; interaction: $F_{8,40}=$ 

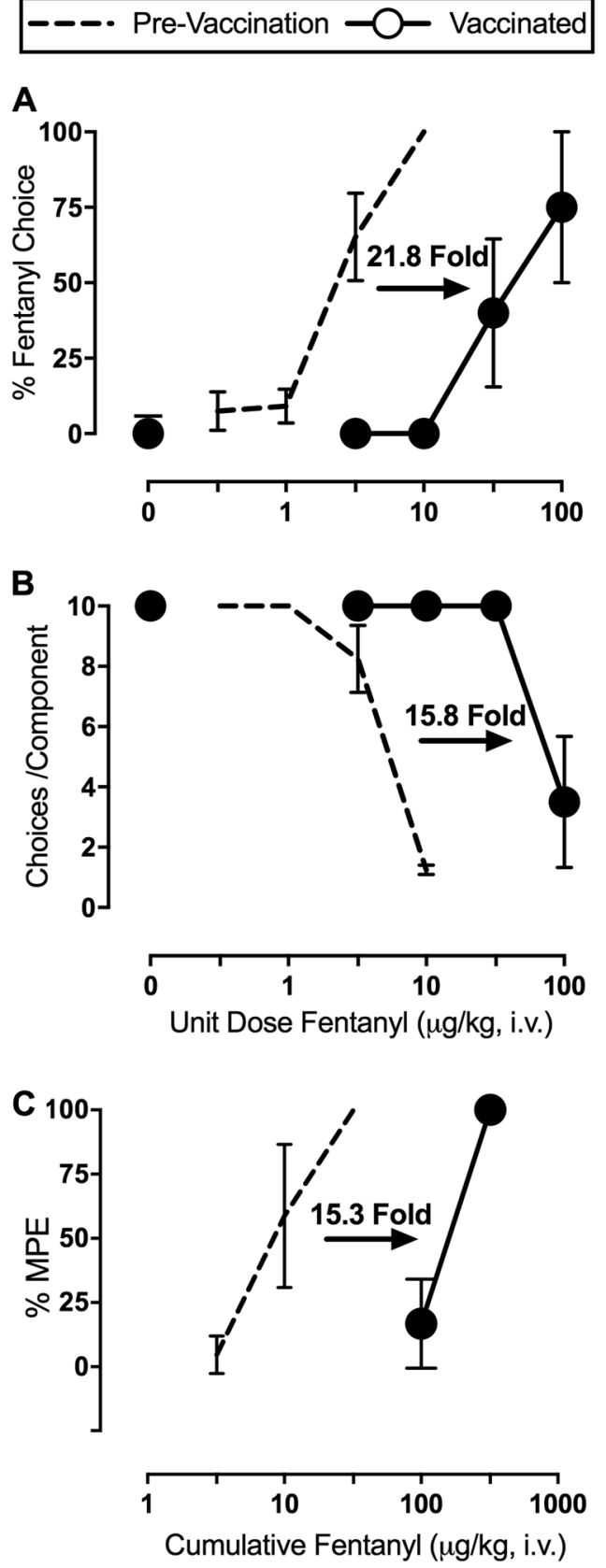

Fig. 4 Surmountability of vaccine effects on fentanyl-vs.-food choice when the unit fentanyl dose was increased 10-fold. Abscissa: intravenous fentanyl dose in $\mu \mathrm{g} / \mathrm{kg} /$ infusion (a, b: unit dose; c: cumulative dose). a ordinate: Percentage of completed ratio requirements on the fentanyl-associated lever. Panel B ordinate: Number of choices completed per component. c ordinate: Percent maximum possible effect (\%MPE) collected in an assay of thermal antinociception. Dashed line represents pre-vaccination results (fentanyl dose range $0.32-10 \mu \mathrm{g} / \mathrm{kg} /$ infusion) and the solid line represents week 21 of the vaccination schedule (fentanyl dose range $10-100 \mu \mathrm{g} / \mathrm{kg} /$ infusion) in the 4 rats that maintained catheter patency ( $n=2$ males, 2 females). Points represent mean \pm SEM and filled symbols denote significant difference relative to prevaccinated data, defined as non-overlapping $95 \%$ confidence limits for $\mathrm{ED}_{50}$ values

12.2, $p<0.001)$. Extended fentanyl access also decreased choices completed during early choice components (1-4) in vaccinated rats (3E: condition: $F_{2,6}=10.7, p=0.01$; interaction: $F_{8,24}=3.2$, $p=0.012$ ).
Surmountability of vaccine effectiveness in fentanyl-vs.-food choice

Figure 4 shows vaccine effectiveness when the unit fentanyl dose available during the fentanyl-vs.-food choice session was increased 10-fold. Relative to pre-vaccination data, a 21.8-fold potency shift in fentanyl choice was observed (4A: baseline $E D_{50}$ (95\% CL): $2.2 \mu \mathrm{g} / \mathrm{kg} / \mathrm{inf}$ (1.2-3.6), week $21 \mathrm{ED}_{50}: 48.0 \mu \mathrm{g} / \mathrm{kg} / \mathrm{inf}$ (18.1-127.4)). Similarly, a 15.8-fold shift in the rate-decreasing effects of fentanyl was observed (4B: baseline $E_{50}: 5.2 \mu \mathrm{g} / \mathrm{kg} / \mathrm{inf}$ (4.0-6.8), week $\left.21 \mathrm{ED}_{50}: 82.0 \mu \mathrm{g} / \mathrm{kg} / \mathrm{inf}(47.2-142.6)\right)$. Finally, a 15.3fold shift in fentanyl antinociceptive potency was observed (Fig. 4c: baseline $\mathrm{ED}_{50}: 8.3 \mu \mathrm{g} / \mathrm{kg}$ (4.5-15.2), week $21 \mathrm{ED}_{50}$ : $127.2 \mu \mathrm{g} / \mathrm{kg}(74.3-217.7))$.

\section{DISCUSSION}

The present study determined the effectiveness of a fentanyl-TT conjugate vaccine on fentanyl self-administration in male and female rats over the course of 21 consecutive weeks. There were three main findings. First, vaccine administration essentially eliminated fentanyl choice and reciprocally increased food choice by week 4 of the vaccine schedule in all rats. Furthermore, vaccine effectiveness was as effective as 7-day treatment with the current FDA-approved OUD pharmacotherapy naltrexone. Moreover, a sustained reduction in fentanyl vs. food choice was demonstrated for 15 weeks in response to only two vaccinations. Second, vaccine administration blunted fentanyl self-administration during 12-h extended fentanyl self-administration sessions and prevented the expression of opioid withdrawal-induced increases in fentanyl-vs.-food choice. Lastly, vaccine effectiveness to decrease fentanyl-vs.-food choice was only surmounted by increasing the unit fentanyl dose 10 -fold, revealing an approximately 22 -fold shift in fentanyl potency. Overall, these preclinical results support the further consideration of this conjugate fentanyl- $T$ vaccine to address the current opioid crisis.

The present results using a within-session IV opioid-vs.-food choice procedure are consistent with previous heroin-vs.-food and remifentanil-vs.-food choice studies in nonhuman primates $[31,32,40]$ and extend these previous findings to the MOR agonist fentanyl and to rats. Continuous 7-day naltrexone treatment produced dose-dependent decreases in fentanyl vs. food choice and dose-dependent rightward shifts in the fentanyl antinociception dose-effect function. The present naltrexone results are consistent with and extend previous findings with acute naltrexone [40] and continuous naloxone treatment [31] on opioid-vs.-food choice in rhesus monkeys. More importantly, these results are consistent with depot naltrexone treatment effects on heroin-vs.-money choice in humans [21] and clinical trial effectiveness [41]. In contrast to naltrexone, continuous 7day clonidine treatment increased fentanyl-vs.-food choice and failed to alter fentanyl antinociception. The present clonidine results are consistent with clonidine as a monotherapy failing to decrease opioid use in a clinical trial [26]. In summary, the present naltrexone and clonidine results provide an appropriate empirical framework with both positive (naltrexone) and negative (clonidine) controls to critically evaluate candidate pharmacotherapies for OUD [42], such as a conjugate fentanyl vaccine.

Two vaccine doses were sufficient to produce a robust decrease in fentanyl-vs.-food choice that was similar in magnitude to naltrexone and sustained for 15 weeks. The present results are consistent with previous studies examining the effectiveness of immunopharmacotherapies to attenuate opioid reinforcement $[12-15,43,44]$. However, these results also extend upon these previous findings in three translationally important ways. First, these results demonstrate that an immunopharmacotherapy can result in increased choice of a non-drug reinforcer (i.e., liquid food) over the target opioid (i.e., fentanyl). This may be of particular 
translational relevance, given that 6 of the 11 diagnostic criteria for OUD are related to the maladaptive allocation of behavior towards the procurement and use of opioids at the expense of non-drug alternative reinforcers (e.g., gainful employment, health, inter-personal relationships; [45]). Accordingly, treatment goals for OUD include not only decreasing behavior maintained by opioids but also increasing behavior maintained by non-drug reinforcers $[6,16]$.

Second, the present study is the first to utilize experimental parameters sufficient to detect a parallel rightward shift in an opioid reinforcement dose-response function following immunopharmacotherapy administration. A vaccine effect that was only detected after the unit IV fentanyl dose range was increased ten-fold. This demonstration of surmountability (i.e., 22-fold shift) supports the interpretation that anti-fentanyl antibodies provided a degree of fentanyl blockade sufficient to blunt fentanyl reinforcement until the anti-fentanyl antibodies became saturated, after which fentanyl was able to activate brain reinforcement circuitry. When considered alongside the positive correlation demonstrated between midpoint titer levels and the fentanyl antinociceptive potency shifts throughout the experimental period, it appears that the maintenance of sufficiently high levels of antibodies will be a critical determinant of translationally relevant reductions in fentanyl reinforcement. By demonstrating that a vaccine booster given over three months after the second priming infusion recapitulated the peak antibody response, these results suggest that vaccine efficacy could be well maintained with a clinicallyfeasible, quarterly dosing regimen.

Lastly, vaccine administration decreased rates of fentanyl selfadministration during 12-h extended fentanyl access sessions and blocked the expression of opioid withdrawal-induced increases in fentanyl-vs.-food choice. The expression of opioid withdrawalinduced increases in fentanyl-vs.-food choice in nonvaccinated rats following an abstinence period, including responding on the opioid-associated lever even when no opioid was available, was consistent with previous studies examining opioid-vs.-food choice in monkeys [28, 31, 32, 46] and rats [29]. Opioid dependence and subsequent withdrawal-induced increases in opioid reinforcement has been proposed as one critical component in the development and expression of OUD [30]. The present results demonstrate that immunopharmacotherapy administration may have the potential to prevent the development and expression of opioid dependence and subsequent withdrawal-induced increases in opioid reinforcement.

Although the present results provide compelling and encouraging evidence for the therapeutic potential of opioidtargeted vaccines, the unique challenges posed by immunopharmacotherapies in the treatment of OUD should also be considered. Three will be mentioned. First, the effectiveness of an immunopharmacotherapy is dependent upon two factors (1) the production of sufficiently high levels of viable titers by the immunized subject's immune system and (2) the antibody affinity for the drug. This latter factor is critical because fentanyl's affinity for the MOR is in the low nanomolar range. This suggests that, unlike current FDA-approved OUD treatments that directly target opioid receptors, responsiveness to opioid-targeted vaccine administration will be dependent on the individual's ability to generate a sufficient immune response to the drug and an immunocompromised individual may not be a suitable patient for drug-targeted immunopharmacotherapy. Second, the onset of peak vaccine effectiveness from the first vaccine administration was on the order of weeks. This lag time could leave subjects vulnerable to the effects of the targeted opioid during this vaccine induction period. One strategy for mitigating this vulnerability could be to combine a vaccine with depot naltrexone, buprenorphine, and/or an anti-drug monoclonal antibody during the vaccine induction period. Finally, because antibodies elicited by opioid-targeted vaccines have a high degree of specificity, an individual could circumvent vaccine effects by misusing a structurally dissimilar opioid. For example, the fentanyl vaccine utilized in the present studies has been shown to exhibit "pan-specificity" towards a variety of fentanyl analogues, including a-methylfentanyl, cis-3-methylfentanyl, and carfentanil $[9,39]$. Yet, this same fentanyl vaccine elicited antibodies displaying very weak affinity towards structurally dissimilar opioids such as methadone and oxycodone $[9,39]$. Accordingly, recent preclinical research has explored the development of combination immunopharmacotherapy approaches directed at multiple, structurally dissimilar abused opioids (e.g., fentanyl and heroin) [47]. Furthermore, antibody specificity elicited by a combination opioid-targeted vaccine would also maintain the flexibility of a patient being treated with a structurally dissimilar opioid (e.g. oxycodone) for pain management. In conclusion, opioid-targeted vaccines may provide for a unique clinically effective option for OUD treatment and the present preclinical evidence supports their continued development and evaluation in human laboratory drug-vs.-money choice studies and clinical trials.

\section{FUNDING AND DISCLOSURE}

Research was supported by institutional professional development funds, the International Study Group Investigating Drugs as Reinforcers (ISGIDAR) travel award, and the National Institute on Drug Abuse of the National Institutes of Health under Award Numbers UH3DA041146, T32DA007027, F32DA047026, and F32AI126628. The National Institute on Drug Abuse had no role in study design, collection, analysis or interpretation of the data, in the writing or decision to submit the manuscript for publication. The manuscript content is solely the responsibility of the authors and does not necessarily reflect the official views of the National Institutes of Health. All authors declare their research has been funded by the $\mathrm{NIH}$. The authors declare no competing interests. KDJ is an inventor on a Scripps Research patent that covers the conjugate fentanyl- $T$ vaccine and has been licensed.

\section{ACKNOWLEDGEMENTS}

We appreciate comments from Dr. Steve Negus during the design of these experiments. We also acknowledge Kevin Costa for writing the original version of the behavioral program that was modified for the choice studies conducted in this manuscript.

\section{ADDITIONAL INFORMATION}

Supplementary Information accompanies this paper at (https://doi.org/10.1038/ s41386-019-0385-9).

Publisher's note: Springer Nature remains neutral with regard to jurisdictional claims in published maps and institutional affiliations.

\section{REFERENCES}

1. Shield BCB 2018. https://www.bcbs.com/the-health-of-america/reports/theopioid-epidemic-america-update.

2. Hedegaard H, Warner M, Minino AM Drug Overdose Deaths in the United States, 1999-2016. NCHS Data Brief. 2017:1-8.

3. Volkow ND, Frieden TR, Hyde PS, Cha SS. Medication-assisted therapies tackling the opioid-overdose epidemic. New Engl J Med. 2014;370:2063-66.

4. Volkow ND, Woodcock J, Compton WM, Throckmorton DC, Skolnick P, Hertz S. et al. Medication development in opioid addiction: Meaningful clinical end points. Sci Transl Med. 2018;10:eaan2595.

5. Volkow ND, Collins FS. The role of science in addressing the opioid crisis. New Engl J Med. 2017;377:391-94.

6. Banks ML, Olson ME, Janda KD. Immunopharmacotherapies for treating opioid use disorder. Trends Pharmacol Sci. 2018;39:908-11. 
7. Baehr C, Pravetoni M. Vaccines to treat opioid use disorders and to reduce opioid overdoses. Neuropsychopharmacology. 2019;44:217-18.

8. Bremer PT, Janda KD. Conjugate vaccine immunotherapy for substance use disorder. Pharmacol Rev. 2017;69:298-315.

9. Hwang CS, Bremer PT, Wenthur CJ, Ho SO, Chiang S, Ellis B, et al. Enhancing efficacy and stability of an antiheroin vaccine: examination of antinociception, opioid binding profile, and lethality. Mol Pharm. 2018;15:1062-72.

10. Sulima A, Jalah R, Antoline JFG, Torres OB, Imler GH, Deschamps JR, et al. A stable heroin analogue that can serve as a vaccine hapten to induce antibodies that block the effects of heroin and its metabolites in rodents and that cross-react immunologically with related drugs of abuse. J Med Chem. 2018;61:329-43.

11. Bremer PT, Schlosburg JE, Banks ML, Steele FF, Zhou B, Poklis JL, et al. Development of a clinically viable heroin vaccine. J Am Chem Soc. 2017;139:8601-11.

12. Bonese KF, Wainer BH, Fitch FW, Rothberg RM, Schuster CR. Changes in heroin self-administration by a rhesus monkey after morphine immunisation. Nature. 1974;252:708-10.

13. Schlosburg JE, Vendruscolo LF, Bremer PT, Lockner JW, Wade CL, Nunes AAK et al. Dynamic vaccine blocks relapse to compulsive intake of heroin. Proc Natl Acad Sci USA. 2013;110:9036-41.

14. Nguyen JD, Hwang CS, Grant Y, Janda KD, Taffe MA. Prophylactic vaccination protects against the development of oxycodone self-administration. Neuropharmacology. 2018;138:292-303.

15. Pravetoni M, Pentel PR, Potter DN, Chartoff EH, Tally L, LeSage MG. Effects of an oxycodone conjugate vaccine on oxycodone self-administration and oxycodoneinduced brain gene expression in rats. PLOS ONE. 2014;9:e101807.

16. Banks ML, Negus SS. Insights from preclinical choice models on treating drug addiction. Trends Pharm Sci. 2017;38:181-94.

17. Epstein $\mathrm{DH}$, Heilig $M$, Shaham $Y$. Science-based actions can help address the opioid crisis. Trends Pharmacol Sci. 2018;39:911-16.

18. Banks ML. Utility of preclinical drug versus food choice procedures to evaluate candidate medications for methamphetamine use disorder. Ann New Y Acad Sci. 2017;1394:92-105.

19. Banks ML, Hutsell BA, Schwienteck KL, Negus SS. Use of preclinical drug vs. food choice procedures to evaluate candidate medications for cocaine addiction. Curr Treat Options. Psychiatry. 2015;2:136-50.

20. Negus SS, Banks ML. Medications Development for Opioid Abuse. In: Pierce RC, Kenny PJ, editors. Addiction. New York: Cold Springs Harbor; 2013.

21. Sullivan MA, Vosburg SK, Comer SD. Depot naltrexone: antagonism of the reinforcing, subjective, and physiological effects of heroin. Psychopharmacology. 2006;189:37-46.

22. Tanum L, Solli K, Latif $Z$, et al. Effectiveness of injectable extended-release naltrexone vs daily buprenorphine-naloxone for opioid dependence: a randomized clinical noninferiority trial. JAMA Psychiatry. 2017;74:1197-205.

23. Mantsch JR, Baker DA, Funk D, Le AD, Shaham Y. Stress-induced reinstatement of drug seeking: 20 years of progress. Neuropsychopharmacology. 2016;41:335-56.

24. Kowalczyk WJ, Phillips KA, Jobes ML, Kennedy AP, Ghitza UE, Agage DA, et al. Clonidine maintenance prolongs opioid abstinence and decouples stress from craving in daily life: a randomized controlled trial with ecological momentary assessment. Am J Psychiatry. 2015;172:760-7.

25. Kowalczyk WJ, Bertz JW, Moran LM, Phillips KA, Ghitza UE, Epstein DH, et al. Clonidine increases the likelihood that abstinence can withstand unstructured time in buprenorphine-maintained outpatients. J Addict Med. 2017;11:454-60.

26. Ling W, Amass L, Shoptaw S, Annon JJ, Hillhouse M, Babcock D, et al. A multicenter randomized trial of buprenorphine-naloxone versus clonidine for opioid, detoxification: findings from the National Institute on Drug Abuse Clinical Trials Network. Addiction. 2005;100:1090-100.

27. Wade $\mathrm{CL}$, Vendruscolo LF, Schlosburg JE, Hernandez DO, Koob GF. Compulsivelike responding for opioid analgesics in rats with extended access. Neuropsychopharmacology. 2015;40:421-28.
28. Wurster RM, Griffiths RR, Findley JD, Brady JV. Reduction of heroin selfadministration in baboons by manipulation of behavioral and pharmacological conditions. Pharmacol Biochem Behav. 1977;7:519-28.

29. Lenoir M, Cantin L, Vanhille N, Serre F, Ahmed SH. Extended heroin access increases heroin choices over a potent nondrug alternative. Neuropsychopharmacology. 2013;38:1209-20.

30. Negus SS, Banks ML. Modulation of drug choice by extended drug access and withdrawal in rhesus monkeys: implications for negative reinforcement as a driver of addiction and target for medications development. Pharmacol Biochem Behav. 2018;164:32-39.

31. Negus SS. Choice between heroin and food in nondependent and heroindependent rhesus monkeys: effects of naloxone, buprenorphine, and methadone. J Pharm Exp Ther. 2006:317:711-23.

32. Negus SS, Rice KC. Mechanisms of withdrawal-associated increases in heroin selfadministration: pharmacologic modulation of heroin vs food choice in heroindependent rhesus monkeys. Neuropsychopharmacology. 2009;34:899-911.

33. Huskinson SL, Naylor JE, Townsend EA, Rowlett JK, Blough BE, Freeman KB. Selfadministration and behavioral economics of second-generation synthetic cathinones in male rats. Psychopharmacology. 2017;234:589-98.

34. Townsend EA, Naylor JE, Negus SS, Edwards SR, Qureshi HN, McLendon HW, et al. Effects of nalfurafine on the reinforcing, thermal antinociceptive, and respiratorydepressant effects of oxycodone: modeling an abuse-deterrent opioid analgesic in rats. Psychopharmacology. 2017;234:2597-605.

35. Thomsen M, Barrett AC, Negus SS, Caine SB. Cocaine versus food choice procedure in rats: environmental manipulations and effects of amphetamine. J Exp Anal Behav. 2013;99:211-33.

36. Negus SS. Rapid assessment of choice between cocaine and food in rhesus monkeys: effects of environmental manipulations and treatment with dAmphetamine and flupenthixol. Neuropsychopharmacology. 2003;28:919-31.

37. Banks ML, Blough BE. Effects of environmental maniuplations and bupropion and risperidone treatments on choice between methamphetamine and food in rhesus monkeys. Neuropsychoharmacology. 2015;40:2198-206.

38. Ossipov MH, Harris S, Lloyd P, Messineo E, Lin BS, Bagley J. Antinociceptive interaction between opioids and medetomidine: systemic additivity and spinal synergy. Anesthesiology. 1990;73:1227-35.

39. Bremer PT, Kimishima A, Schlosburg JE, Zhou B, Collins KC, Janda KD. Combatting synthetic designer opioids: a conjugate vaccine ablates lethal doses of fentanyl class drugs. Angew Chem Int Ed. 2016;55:3772-75.

40. Maguire DR, Gerak LR, Woods JH, Husbands SM, Disney A, France CP. Longlasting effects of methocinnamox on opioid self-administration in rhesus monkeys. J Pharmacol Exp Ther. 2019;368:88-99.

41. Comer SD, Sullivan MA, Yu E, et al. Injectable, sustained-release naltrexone for the treatment of opioid dependence: a randomized, placebo-controlled trial. Arch Gen Psychiatry. 2006;63:210-18.

42. Matthew L, Banks E, Andrew T, Negus SS. Testing the 10 most wanted: a preclinical algorithm to screen candidate opioid use disorder medications. Neuropsychopharmacology. 2019;44:1011-12.

43. Stowe GN, Vendruscolo LF, Edwards S, Schlosburg JE, Misra KK, Schulteis G, et al. A vaccine strategy that induces protective immunity against heroin. J Med Chem. 2011;54:5195-204.

44. Raleigh MD, Pentel PR, LeSage MG. Pharmacokinetic correlates of the effects of a heroin vaccine on heroin self-administration in rats. PLOS ONE. 2014;9:e115696.

45. Association AP. Diagnositc and Statistical Manual of Mental Disorders. Fifth Edition. Arlington, VA: American Psychiatric Association; 2013.

46. Spragg SDS. Morphine Addiction in Chimpanzees. Comparative Psychology Monographs. Baltimore, MD: The John Hopkins Press; 1940. p. 1-132.

47. Hwang CS, Smith LC, Natori Y, Ellis B, Zhou B, Janda KD. Efficacious vaccine against heroin contaminated with fentanyl. ACS chemical. Neuroscience. 2018;9:1269-75. 